\title{
Rational Dyck Paths in the Non Relatively Prime Case
}

\author{
Eugene Gorsky ${ }^{12}$, Mikhail Mazin ${ }^{3}$, and Monica Vazirani ${ }^{1}$ \\ ${ }^{1}$ University of California at Davis, Davis, California, US \\ ${ }^{2}$ International Laboratory of Representation Theory and Mathematical Physics, NRU-HSE, Moscow, Russia \\ ${ }^{3}$ Kansas State University, Manhattan, Kansas, US
}

\begin{abstract}
We study the relationship between rational slope Dyck paths and invariant subsets in $\mathbb{Z}$, extending the work of the first two authors in the relatively prime case. We also find a bijection between $(d n, d m)-$ Dyck paths and $d$-tuples of $(n, m)$-Dyck paths endowed with certain gluing data. These are first steps towards understanding the relationship between the rational slope Catalan combinatorics in non relatively prime case and the geometry of affine Springer fibers and representation theory.

Résumé. Nous étudions les relations entre les chemins de Dyck à pente rationnelle et des sous-ensembles invariants en $\mathbb{Z}$, étendant le travail des deux premiers auteurs dans le cas relativement premier. On trouve aussi une bijection entre les $(d n, d m)$-chemins de Dyck et des $d$-tuples de $(n, m)$-chemins de Dyck avec certaines données de collage. Ce sont les premiers pas vers la compréhension des relations entre la combinatoire Catalan de pentes rationnelles dans le cas non-relativement premier, et la géométrie des fibres affines de Springer et la théorie de la représentation.
\end{abstract}

Keywords. rational Dyck paths, rational Catalan combinatorics, simultaneous core partitions, invariant integer subsets, semigroups

\section{Introduction}

Catalan numbers, in one of their incarnations, count the number of Dyck paths, that is, the lattice paths in a square which never cross the diagonal. In recent years, a number of interesting results and conjectures [3, 5, 6, 7, 8, 9, 17] about "rational Catalan combinatorics" have been formulated. An $(n, m)$-Dyck path is a lattice path in the $n \times m$ rectangle which never crosses the diagonal. We will denote the set of all $(n, m)$-Dyck paths by $Y_{n, m}$. For coprime $m$ and $n$ there are a number of interesting maps involving $Y_{n, m}$ :

(a) J. Anderson constructed a bijection $\mathcal{A}$ between $Y_{n, m}$ and the set $\operatorname{Core}_{n, m}$ of simultaneous $(n, m)$ core partitions.

(b) Armstrong, Loehr and Warrington defined a "sweep" map $\zeta: Y_{n, m} \rightarrow Y_{n, m}$ and conjectured that it is bijective. 
(c) The first two authors defined two maps $\mathcal{D}$ and $\mathcal{G}$ between $Y_{n, m}$ and the set $\mathbf{M}_{n, m}$ of $(+n,+m)$ invariant subsets of $\mathbb{Z}_{\geq 0}$. If combined with a natural bijection between Core ${ }_{n, m}$ and $\mathbf{M}_{n, m}$, the map $\mathcal{D}$ coincides with $\mathcal{A}$. Furthermore, one can prove that $\zeta=\mathcal{G} \circ \mathcal{D}^{-1}$.

Remark 0.1 Recently, after the original version of this manuscript was submitted, Nathan Williams posted a preprint [16] containing a proof of the bijectivity of the sweep map. Statements below involving $\zeta$ should be modified appropriately.

We illustrate all these maps in Figure 1: bidirected arrows indicate bijections, and directed arrows indicate maps for which the inverse is not known in general.

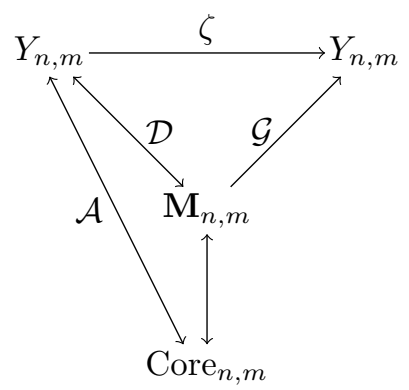

Fig. 1: Rational Catalan maps in the coprime case

The goal of the present paper is a partial generalization of Figure 1 to the non-coprime case. Let $(n, m)$ be relatively prime, and $d$ be a positive integer. Let $N=d n$ and $M=d n$. The set $Y_{N, M}$ is well defined for all $n, m, d$, and the definition of $\zeta$ can be carried over with minimal changes. However, the sets Core $_{N, M}$ and $\mathbf{M}_{N, M}$ become infinite. Indeed, an $(+N,+M)$-invariant subset of $\mathbb{Z}_{\geq 0}$ can be identified with a collection of $d(+n,+m)$-invariant subsets in each remainder mod $d$. These subsets can be arbitrarily shifted with respect to each other. More abstractly, this defines a map $\pi: \mathbf{M}_{N, M} \rightarrow\left(\mathbf{M}_{n, m}\right)^{d}$ and different shifts correspond to different preimages under $\pi$.

To resolve this problem, we introduce a certain equivalence relation $\sim$ on $\mathbf{M}_{N, M}$. If $\Delta_{1} \sim \Delta_{2}$ then $\pi\left(\Delta_{1}\right)=\pi\left(\Delta_{2}\right)$, so $\pi$ is well defined on $\mathbf{M}_{N, M} / \sim$. The following theorem is the main result of the paper.

Theorem 0.2 For all positive $N, M$ one can define maps

$$
\mathcal{D}, \mathcal{G}: \mathbf{M}_{N, M} / \sim \longrightarrow Y_{N, M}
$$

such that the following results hold:

(a) The map $\mathcal{D}$ is a bijection.

(b) The "sweep" map factorizes similarly to the coprime case: $\zeta=\mathcal{G} \circ \mathcal{D}^{-1}$.

(c) Let $d=\operatorname{gcd}(N, M), n=N / d$, and $m=M / d$. The composition

$$
\operatorname{col}_{d}:=\mathcal{D}^{d} \circ \pi \circ \mathcal{D}^{-1}: Y_{N, M} \rightarrow\left(Y_{n, m}\right)^{d},
$$


can be described as following: the $N+M$ steps in an $(N, M)$-Dyck path are colored in $d$ colors, i.e. by $\mathbb{Z} / d \mathbb{Z}$, such that there are $n+m$ steps of the same color $i$, and these steps will form an $(n, m)$-Dyck path after possibly shifting by integer multiples of $\overrightarrow{(m,-n)}$ to make them connected.

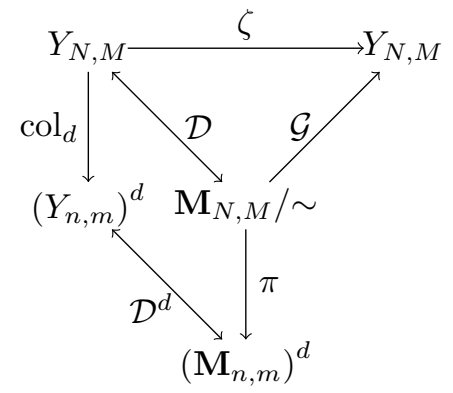

Fig. 2: Rational Catalan maps in the non-coprime case

We illustrate all these maps in Figure 2. We also give an explicit description of the "coloring map" $\operatorname{col}_{d}$. In the "classical" case $M=N$ we get $d=N$ and $m=n=1$, therefore $\operatorname{col}_{d}$ colors a Dyck path in $n$ colors such that the pairs of steps of the same color form an $(1,1)$ Dyck path. We prove the following:

Corollary 0.3 Present a Dyck path as a regular sequence of parentheses. Then every opening and its corresponding closing parenthesis have the same color under $\mathrm{col}_{d}$.

\section{Relatively prime case}

Let $(n, m)$ be a pair of relatively prime positive integers. Consider an $n \times m$ rectangle $R_{n, m}$. Let $Y_{n, m}$ be the set of Young diagrams that fit under the diagonal in $R_{n, m}$. We will often abuse notation by identifying a diagram $D \in Y_{n, m}$ with its boundary path (sometimes also called a rational Dyck path), and with the corresponding partition. We will also think about the rectangle $R_{n, m}$ as a set of boxes, identified with a subset in $\mathbb{Z}_{\geq 0}$ with the bottom-left corner box identified with $(0,0)$. In our convention, the boundary path of $D \subset R_{n, m}$ follows the boundary from top-left corner $(0, n)$ to the bottom-right corner $(m, 0)$. We will refer to the direction along the boundary path from $(0, n)$ toward $(m, 0)$ as from higher to lower. See Example 1.11 below.

There are two important combinatorial statistics on the set $Y_{n, m}$ : area and dinv .

Definition 1.1 Let $D \in Y_{n, m}$. Then area $(D)$ is equal to the number of whole boxes that fit between the diagonal of $R_{n, m}$ and the boundary path of $D$.

Note that area $(D)$ ranges from 0 for the full diagram to $\delta=\frac{(m-1)(n-1)}{2}$ for the empty diagram. The co-area $(D)=\delta-\operatorname{area}(D)$ is then just the number of boxes in the Young diagram $D$. One natural approach to the dinv statistic is to define the $\operatorname{map} \zeta: Y_{n, m} \rightarrow Y_{n, m}$ and then set $\operatorname{dinv}(D):=\operatorname{area}(\zeta(D))$. In the case $m=n+1$ the map $\zeta$ was first defined by Haglund ([10]), then it was generalized by Loehr to the case $m=k n+1$ for any $k \in \mathbb{Z}_{\geq 0}([11])$, and to the general case of any relatively prime $(n, m)$ by 
Gorsky and Mazin in [7]. In [3] it was put into even larger framework of so called sweep maps. Below is one of the possible equivalent definitions.

Definition 1.2 The rank of a box $(x, y) \in \mathbb{Z}^{2}$ is given by the linear function

$$
\ell(x, y)=m n-m-n-n x-m y .
$$

Note that the boxes of non-negative ranks are exactly those that fit under the main diagonal of $R_{n, m}$. Let $D \in Y_{n, m}$. One ranks the steps of the boundary path of $D$ as follows.

Definition 1.3 The rank of a vertical step of $D$ equals to the rank of the box immediately to the left of it. The rank of a horizontal step equals to the rank of the box immediately above it.

In other words, the ranks of steps can be defined inductively as follows. We follow the boundary path of $D$ starting from the top-left corner. The first step is ranked 0 . Otherwise, the rank of every horizontal step equals the rank of the previous step minus $n$, and the rank of every vertical step equals the rank of the previous step plus $m$.

Note that for relatively prime $(n, m)$ all the ranks of the steps of a diagram $D \in Y_{n, m}$ are distinct.

Definition 1.4 The boundary path of the diagram $\zeta(D)$ is obtained from the boundary path of $D$ by rearranging the steps in the decreasing order of ranks.

The definition of the map $\zeta$ is illustrated in Example 1.11 .

One can verify that the diagram $\zeta(D)$ fits under the diagonal of $R_{n, m}$ (see [7] and [3]). It was conjectured that the map $\zeta$ is a bijection. See Remark 0.1 . Some partial progress was made by Haglund, Loehr, Gorsky, and Mazin:

Theorem 1.5 ([10, 11, []]) The map $\zeta: Y_{n, m} \rightarrow Y_{n, m}$ is a bijection for $m=k n \pm 1$ for any $k \in \mathbb{Z}_{\geq 0}$.

Bijectivity of $\zeta$ in the general case has attracted a lot of attention: see [17] and [6]. Most recently, Nathan Williams proved bijectivity of $\zeta$ for general coprime $n, m$ ([16]). The following approach to studying $\zeta$ was suggested in [7].

Definition 1.6 Let $\mathbf{M}_{n, m}$ be the set of subsets $\Delta \subset \mathbb{Z}_{\geq 0}$ such that $\Delta+m \subset \Delta, \Delta+n \subset \Delta$, and $\min (\Delta)=0$. We say $\Delta \in \mathbf{M}_{n, m}$ is $(+n,+m)$-invariant.

In [7] two maps $\mathcal{D}$ and $\mathcal{G}$ from the set $\mathbf{M}_{n, m}$ to $Y_{n, m}$ were constructed. Let $\Delta \in \mathbf{M}_{n, m}$.

Definition 1.7 The diagram $\mathcal{D}(\Delta)$ consists of all boxes that fit under the diagonal in $R_{n, m}$, and whose ranks belong to $\Delta$.

In particular, one gets that $\mathcal{D}\left(\Gamma_{n, m}\right)=\emptyset$, where $\Gamma_{n, m}:=\left\{a n+b m \mid a, b \in \mathbb{Z}_{\geq 0}\right\}$ is the semigroup generated by $m$ and $n$, and $\mathcal{D}\left(\mathbb{Z}_{\geq 0}\right)$ is the full diagram containing all the boxes below the diagonal. Note that the $(+n,+m)$-invariance of $\Delta$ implies that $\mathcal{D}(\Delta)$ is indeed a Young diagram. Note also that $\mathcal{D}$ is a bijection. Indeed, it is not hard to see that rank provides a bijection between the boxes below the diagonal in $R_{n, m}$ and the integers in $\mathbb{Z}_{\geq 0} \backslash \Gamma_{n, m}$.

Definition 1.8 The numbers $0=a_{0}<a_{1}<\ldots<a_{n-1}$, such that

$$
\left\{a_{0}, \ldots, a_{n-1}\right\}=\Delta \backslash(\Delta+n)
$$



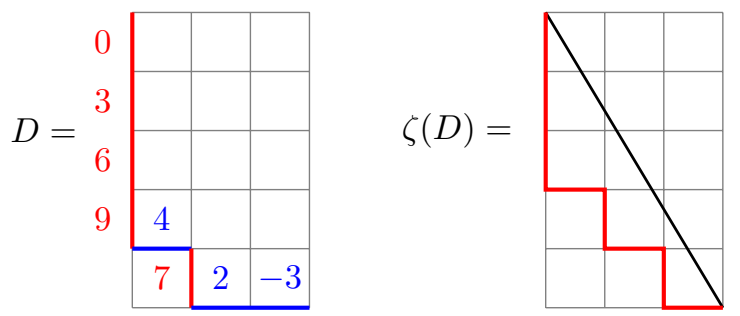

Fig. 3: Here $n=5$ and $m=3$. On the left is the diagram $D=\mathcal{D}(\{0,3,5,6,7,8, \ldots\})$, and on the right is the diagram $\zeta(D)$.

are called the n-generators of $\Delta$. The numbers $\left\{b_{0}<b_{1}<\ldots<b_{m-1}\right\}$ such that

$$
\left\{b_{0}, \ldots, b_{m-1}\right\}=(\Delta-m) \backslash \Delta
$$

are called the m-cogenerators of $\Delta$.

Remark 1.9 Let $D=\mathcal{D}(\Delta)$. Note that the ranks of the vertical steps of $D$ are exactly the $n$-generators of $\Delta$, and the ranks of the horizontal steps of $D$ are exactly the $m$-cogenerators of $\Delta$.

Definition 1.10 The diagram $\mathcal{G}(\Delta)$ has row lengths $g_{0}, \ldots, g_{n-1}$ given by the following formula:

$$
g_{k}=\sharp\left\{b_{i} \mid b_{i}>a_{k}\right\} .
$$

Equivalently, the boundary path of $\mathcal{G}(\Delta)$ is obtained by arranging the set $\left\{a_{0}, \ldots, a_{n-1}, b_{0}, \ldots, b_{m-1}\right\}$ in decreasing order and replacing $n$-generators by vertical steps and $m$-cogenerators by horizontal steps.

It is not hard to conclude from the above definitions that

$$
\zeta(D)=\mathcal{G} \circ \mathcal{D}^{-1}(D)
$$

Example 1.11 For example, if $m=3, n=5$, and $\Delta=\{0,3,5,6,7,8, \ldots\}$ then the 5-generators are $0,3,6,7,9$ and 3-cogenerators are -3,2,4. (Note that the diagram $\mathcal{D}(\Delta)$ consists of just one box, whose rank is 7 .) The ranked boundary path of $D$ is

$$
\begin{array}{cccccccc}
\mathrm{v} & \mathrm{v} & \mathrm{v} & \mathrm{v} & \mathrm{h} & \mathrm{v} & \mathrm{h} & \mathrm{h} \\
0 & 3 & 6 & 9 & 4 & 7 & 2 & -3
\end{array}
$$

which we sort to the boundary path of $\zeta(D)$

$$
\begin{array}{cccccccc}
\mathrm{v} & \mathrm{v} & \mathrm{v} & \mathrm{h} & \mathrm{v} & \mathrm{h} & \mathrm{v} & \mathrm{h} \\
9 & 7 & 6 & 4 & 3 & 2 & 0 & -3
\end{array}
$$

See Figure 3 for the diagrams $D$ and $\zeta(D)$. Note, that if one takes the union of the 5-generators and 3cogenerators and reads them in decreasing order, then one gets $9,7,6,4,3,2,0,-3$. Replacing generators by "v" and cogenerators by " $\mathrm{h}$ ", one gets vvvhvhvh, which is the boundary path of $\zeta(D)$.

The approach with invariant subsets allows one to relate the dinv statistic to certain varieties that appear in algebraic geometry as local versions of compactified Jacobians (see Beauville [4] and Piontkowski [15]), and in representation theory as homogeneous affine Springer fibers, where they were first considered by Lusztig and Smelt in [13] and then by Piontkowski [15]. 


\section{Non-relatively prime case.}

The notion of a rational Dyck path naturally generalizes to the non relatively prime case. Let $(n, m)$ be relatively prime, and $d$ be a positive integer. Let $N=d n$ and $M=d n$. Consider an $N \times M$ rectangle $R_{N, M}$ and the set $Y_{N, M}$ of Young diagrams that fit under the diagonal in $R_{N, M}$. The area statistic can be generalized directly. The dinv statistic and the map $\zeta$ are a bit more tricky. It is convenient to adjust the rank function on the boxes in the following way:

$$
\ell(x, y)=d m n-m-n-n x-m y .
$$

The steps of the boundary path of a diagram $D \in Y_{N, M}$ are ranked as before. However, for $d>1$ some distinct steps might have the same rank, therefore rearranging the steps of the path according to their rank is problematic. The following idea for overcoming this difficulty was suggested by François Bergeron. It also can be found in [3].

Definition 2.1 Let $D \in Y_{N, M}$. The boundary path of the diagram $\zeta(D)$ is obtained from the boundary path of $D$ by rearranging the steps so their ranks are weakly decreasing. If two steps have the same rank, then they are ordered in the reversed order of appearance in the boundary path of D.

Example 2.2 Consider the diagram $D \in Y_{9,6}$ with the boundary path vvvvvhvhhhvvhvh (see Figure 4). The ranked boundary path of $D$ is

$$
\begin{array}{ccccccccccccccc}
\mathrm{v} & \mathrm{V} & \mathbf{V} & \mathrm{V} & \mathrm{V} & \mathrm{h} & \mathrm{v} & \mathbf{h} & \mathbf{h} & \mathrm{h} & \mathrm{v} & \mathrm{v} & \mathrm{h} & \mathbf{v} & \mathrm{h} \\
0 & 2 & 4 & 6 & 8 & 5 & 7 & 4 & 1 & -2 & 0 & 2 & -1 & 1 & -2
\end{array}
$$

which we sort to the boundary path of $\zeta(D)$

$\begin{array}{ccccccccccccccc}\mathrm{V} & \mathrm{v} & \mathrm{v} & \mathrm{h} & \mathbf{h} & \mathbf{v} & \mathrm{v} & \mathrm{v} & \mathbf{v} & \mathbf{h} & \mathrm{v} & \mathrm{v} & \mathrm{h} & \mathrm{h} & \mathrm{h} \\ 8 & 7 & 6 & 5 & 4 & 4 & 2 & 2 & 1 & 1 & 0 & 0 & -1 & -2 & -2\end{array}$.

There are two steps of rank 1 (also of rank 4) marked in bold type to highlight the necessary reordering of their corresponding $\mathbf{v} \mathbf{h}$.

Now the statistic dinv can be defined as

$$
\operatorname{dinv}(D):=\operatorname{area}(\zeta(D)) .
$$

Note that in [5] a different definition of dinv for non relatively prime case is used:

$$
\operatorname{dinv}^{\prime}(D):=\sharp\left\{\square \in D: \frac{\operatorname{leg}(\square)}{\operatorname{arm}(\square)+1}<\frac{n}{m} \leq \frac{\operatorname{leg}(\square)+1}{\operatorname{arm}(\square)}\right\}
$$

Lemma 2.3 One has

$$
\operatorname{dinv}(D)=\operatorname{dinv}^{\prime}(D)
$$

for any $D \in Y_{N, M}$. 

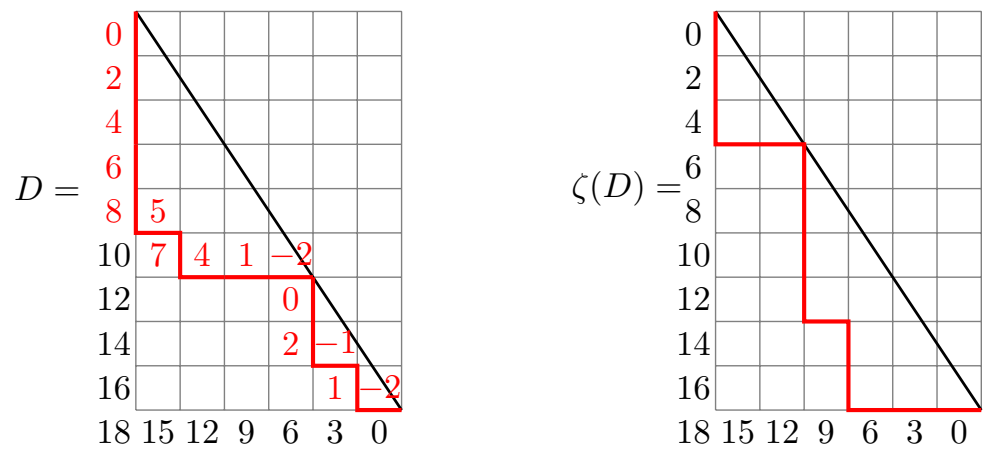

Fig. 4: Here $n=9$ and $m=6$. On the left is the diagram $D$ with the boundary path vvvvvhvhhhvvhvh, and on the right is the diagram $\zeta(D)$.

This result follows from Corollary 1 on page 8 in [14].

The set $\mathbf{M}_{N, M}$ of subsets $0 \in \Delta \subset \mathbb{Z}_{\geq 0}$ that are invariant under addition of $M$ and $N$ is infinite for $\operatorname{gcd}(N, M)=d>1$. Therefore, there is no hope to construct a bijection between the set of such subsets and $Y_{N, M}$. On the other hand, the the map $\mathcal{G}: \mathbf{M}_{N, M} \rightarrow Y_{N, M}$ is still well defined. We instead define an equivalence relation on the invariant subsets, so that the relative order of $N$-generators and $M$-cogenerators is the same within an equivalence class, and then construct a bijection $\mathcal{D}$ between the equivalent classes $\mathbf{M}_{N, M} / \sim$ and $Y_{N, M}$, so that one gets $\zeta=\mathcal{G} \circ \mathcal{D}^{-1}$ as in the $d=1$ case.

Given an $(+N,+M)$-invariant subset $\Delta$ one can extract $d$ many $(+n,+m)$-invariant subsets from it by the following procedure: for each $r \in\{0,1 \ldots, d-1\}$ consider the subset in $\Delta$ consisting of all integers congruent to $r$ modulo $d$, subtract $r$ from all these elements and then divide by $d$. In other words one has

$$
\Delta_{r}=[(\Delta \cap(d \mathbb{Z}+r))-r] / d .
$$

Note that the subsets $\Delta_{r}$ for $r>0$ might be not 0-normalized. Note also that $\Delta$ can be uniquely reconstructed from $\Delta_{0}, \ldots, \Delta_{d-1}$, so we have a bijection between 0 -normalized $(+N,+M)$-invariant subsets and (ordered or $\mathbb{Z} / d \mathbb{Z}$-colored) collections of $d$ many $(+n,+m)$-invariant subsets, such that $\Delta_{0}$ is zero normalized and $\Delta_{i} \subset \mathbb{Z}_{\geq 0}$ for all $i$.

Let us first describe this in an example. The idea of the equivalence relation is that one should fix the collection $\Delta_{0}, \ldots, \Delta_{d-1}$ up to shifts, but allow them to shift with respect to each other if it does not change the total relative order of $N$-generators and $M$-cogenerators of $\Delta$. By a shift of $\Delta$, we mean replacing $\Delta$ with $\Delta+k$ for some $k \in \mathbb{Z}$.

Example 2.4 Let $(M, N)=(4,6)$. The following two elements of $Y_{N, M}$ are equivalent. Let $\Delta^{1}$ be given by:

$\begin{array}{cccccccccccccccc}0 & 1 & 2 & 3 & 4 & 5 & 6 & 7 & 8 & 9 & 10 & 11 & 12 & 13 & 14 & \ldots \\ \times & \circ & \square & \circ & \times & \square & \bullet & \circ & \times & \times & \bullet & \square & \bullet & \times & \bullet & \ldots\end{array}$

and $\Delta^{2}$ : 


$$
\begin{array}{cccccccccccccccl}
0 & 1 & 2 & 3 & 4 & 5 & 6 & 7 & 8 & 9 & 10 & 11 & 12 & 13 & 14 & \ldots \\
\times & \circ & \square & \circ & \times & \circ & \bullet & \square & \times & \circ & \bullet & \times & \bullet & \square & \bullet & \ldots
\end{array}
$$

Here $\times$ 's are 6-generators, $\square$ 's are the 4-cogenerators, $\bullet$ 's are other elements of the subset, and $\circ$ 's are the other elements of the complement. Note that not all 6 generators and 4 cogenerators fit in the pictures. It is more illustrative to split $\Delta^{1}$ into its even and odd parts:

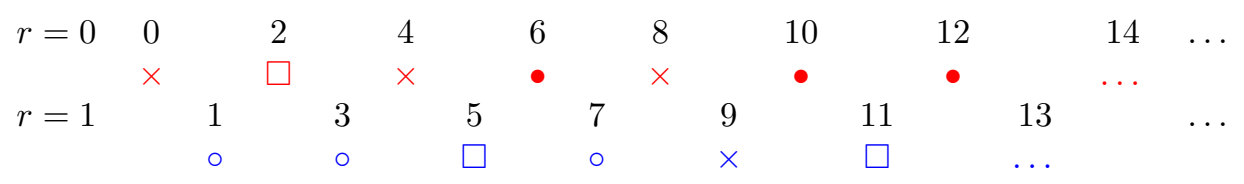

It is more compact to then stack them as

$$
\begin{array}{cccccccccccccc}
r=0 & -4 & -2 & 0 & 2 & 4 & 6 & 8 & 10 & 12 & 14 & 16 & 18 & \ldots \\
& \square & \circ & \times & \square & \times & \bullet & \times & \bullet & \bullet & \bullet & \bullet & \ldots & \\
r=1 & -3 & -1 & 1 & 3 & 5 & 7 & 9 & 11 & 13 & 15 & 17 & 19 & \ldots \\
& \circ & \circ & \circ & \circ & \square & \circ & \times & \square & \times & \bullet & \times & \ldots &
\end{array}
$$

Then we just record $\Delta_{0}^{1}$ and $\Delta_{1}^{1}$ :

$$
\begin{array}{ccccccccccccccc} 
& -2 & -1 & 0 & 1 & 2 & 3 & 4 & 5 & 6 & 7 & 8 & 9 & 10 & \ldots \\
\Delta_{0}^{1} & \square & \circ & \times & \square & \times & \bullet & \times & \bullet & \bullet & \bullet & \bullet & \ldots & & \\
\Delta_{1}^{1} & \circ & \circ & \circ & \circ & \square & \circ & \times & \square & \times & \bullet & \times & \ldots & &
\end{array}
$$

To restore $\Delta^{1}$ one should multiply both $\Delta_{0}^{1}$ and $\Delta_{1}^{1}$ by two, add one to $\Delta_{1}^{1}$, and merge them together. In other words, $\Delta^{1}=2 \Delta_{0}^{1} \cup\left(1+2 \Delta_{1}^{1}\right)$.

Similarly, for $\Delta^{2}$ one gets

$\begin{array}{ccccccccccccccc} & -2 & -1 & 0 & 1 & 2 & 3 & 4 & 5 & 6 & 7 & 8 & 9 & 10 & \ldots \\ \Delta_{0}^{2} & \square & \circ & \times & \square & \times & \bullet & \times & \bullet & \bullet & \bullet & \bullet & \bullet & \ldots & \\ \Delta_{1}^{2} & \circ & \circ & \circ & \circ & \circ & \square & \circ & \times & \square & \times & \bullet & \times & \ldots & \end{array}$

Note that the sequences of $N$-generators and $M$-cogenerators are the same for $\Delta^{1}$ and $\Delta^{2}$, even if we take into account the remainder modulo 2 . In both cases one gets

where red is for even generators and cogenerators $(r=0)$, and blue is for odd $(r=1)$. The reason we set $\Delta^{1} \sim \Delta^{2}$ is that in this example the odd part can be shifted by 1 with respect to the even part without changing the sequence or parity of generators and cogenerators. Note that one cannot shift further: in $\Delta^{1}$ one cannot shift the odd part to the left, and in $\Delta^{2}$ one cannot shift the odd part to the right. Also note that while $\Delta_{0}^{1}=\Delta_{0}^{2}, \Delta_{1}^{1}=-1+\Delta_{1}^{2}$.

The formal definition of the equivalence classes is as follows:

Definition 2.5 Let $\Delta^{1}, \Delta^{2} \in \mathbf{M}_{N, M}$. Let $\left\{\Delta_{0}^{1}, \ldots, \Delta_{d-1}^{1}\right\} \subseteq \mathbf{M}_{n, m}$ and $\left\{\Delta_{0}^{2}, \ldots, \Delta_{d-1}^{2}\right\} \subseteq \mathbf{M}_{n, m}$ be the corresponding collections of $(+n,+m)$-invariant subsets as in (1). We say that $\Delta^{1}$ and $\Delta^{2}$ are equivalent or $\Delta^{1} \sim \Delta^{2}$ if there exist a permutation $\sigma \in S_{d}$ such that for each $i$ there exists an integer $k_{i}$ such that $\Delta_{i}^{1}=k_{i}+\Delta_{\sigma(i)}^{2}$ and the following condition is satisfied. The sequence of $\mathbb{Z} / d \mathbb{Z}$-colored $N$-generators and $M$-cogenerators of $\Delta^{1}$ agrees with the sequence of $\sigma$-twisted colored $N$-generators and $M$-cogenerators of $\Delta^{2}$. 
Remark 2.6 Note that one always has $\sigma(0)=0$, because the minimal $N$-generator is 0 for both $\Delta^{1}$ and $\Delta^{2}$.

Obviously, the order of $N$-generators and $M$-cogenerators is preserved within an equivalence class. The bijection to $Y_{M, N}$ is constructed in two steps. First, we choose the "minimal" representative in each class, and second, we construct a Young diagram for each minimal representative. Bijectivity is evident from the construction.

Definition 2.7 The skeleton of an $(+N,+M)$-invariant subset $\Delta$ is the set consisting of its $N$-generators and $M$-cogenerators.

Note that one can uniquely reconstruct the invariant subset $\Delta$ from its skeleton. Indeed, the skeleton contains all the $N$-generators of $\Delta$, and to distinguish the $N$-generators from the $M$-cogenerators one should simply choose the biggest elements in each congruence class mod $N$.

Example 2.8 The skeleton of $\Delta^{1}$ from Example 2.4 above is $\{-4,0,2,4,5,8,9,11,13,17\}$.

Definition 2.9 Let $\Delta$ be a 0 -normalized $(+N,+M)$-invariant subset and let $\Delta_{0}, \ldots, \Delta_{d-1}$ be the corresponding collection of $(+n,+m)$-invariant subsets as in (1). We say that $\Delta$ is minimal if the following conditions are satisfied:

1. For every $0<i<d$ there exists $j<i$ such that the intersection of the skeletons of $\Delta_{i}$ and $\Delta_{j}$ is nonempty;

2. If the skeleton of $\Delta_{i}$ has empty intersection with the skeleton of $\Delta_{i+1}$ then $\min \left(\Delta_{i}\right)<\min \left(\Delta_{i+1}\right)$.

Theorem 2.10 Every equivalence class of $(+N,+M)$-invariant subsets contains a unique minimal element.

Sketch of the proof. Let $\Delta \in \mathbf{M}_{N, M}$. Consider its skeleton colored with respect to the remainders modulo $d$. Let us allow the parts of the skeleton of different colors to shift with respect to each other while preserving the distances between elements of the skeleton of the same color and not changing the total relative order of generators and co-generators. Let us fix the part of the skeleton with remainder 0 and shift the other colors to the left as much as possible.

Note that as parts of the skeleton of $\Delta$ shift, the correspondence between colors and remainders change. Moreover, it might happen that we will get two different colors in the same remainder. Let $S_{0}, \ldots, S_{d-1} \subset$ $\mathbb{Z}$ be the parts of the shifted skeleton of different colors. Let $r_{i}$ be the remainder of $S_{i}$ modulo $d$. We can require the indices $i$ and the remainders $r_{i}$ to satisfy the following conditions.

1. If $r_{i}<r_{j}$, then $i<j$;

2. If $r_{i}=r_{j}$ and $\min \left(S_{i}\right)<\min \left(S_{j}\right)$, then $i<j$.

Note that these conditions determine the order on colors uniquely. Now, let

$$
S^{\prime}=\bigcup d\left\lfloor\frac{S_{i}}{d}\right\rfloor+i
$$

Then the subset $\Delta^{\prime} \in \mathbf{M}_{N, M}$ is the unique invariant subset with skeleton $S^{\prime}$. In other words, we reassign remainders to colors according to the refined order, while preserving the sets $\left\lfloor\frac{S_{i}}{d}\right\rfloor$ for each color. By construction, we have $\Delta \sim \Delta^{\prime}$. It is also not hard to see that $\Delta^{\prime}$ is minimal. One can also see that if $\Delta$ is minimal, then $\Delta^{\prime}=\Delta$. 
Example 2.11 Let $(M, N)=(6,9)$, so that $d=3$. Let $\Delta$ be such that $\Delta_{1}, \Delta_{2}$, and $\Delta_{3}$ are given by

$\begin{array}{ccccccccccccc} & -2 & -1 & 0 & 1 & 2 & 3 & 4 & 5 & 6 & 7 & 8 & \ldots \\ \Delta_{0} & \square & \circ & \times & \square & \times & \bullet & \times & \bullet & \bullet & \bullet & \bullet & \ldots \\ \Delta_{1} & \square & \square & \times & \times & \times & \bullet & \bullet & \bullet & \bullet & \bullet & \bullet & \ldots \\ \Delta_{2} & \circ & \circ & \circ & \circ & \circ & \circ & \square & \square & \times & \times & \times & \ldots\end{array}$

This subset $\Delta \in \mathbf{M}_{9,6}$ is minimal according to the definition. The skeletons of the $(+2,+3)$-invariant subsets $\Delta_{r}, r=0,1,2$ are $\{-2,0,1,2,4\},\{-2,-1,0,1,2\},\{4,5,6,7,8\}$, respectively. Indeed, the skeleton of $\Delta_{0}$ intersects both the skeleton of $\Delta_{1}$ and the skeleton of $\Delta_{2}$, while the skeletons of $\Delta_{1}$ and $\Delta_{2}$ do not intersect; one has $\min \left(\Delta_{1}\right)=0<\min \left(\Delta_{2}\right)=6$. However, if we let corresponding parts of the skeleton of $\Delta$ shift as far left as possible, then both blue and green parts will end up in remainder 1.

The last step in proving the main result is to construct the Young diagram $\mathcal{D}(\Delta) \in Y_{N, M}$ from a minimal $(+N,+M)$-invariant subset $\Delta$. The construction goes as follows. Consider the $(+n,+m)$ invariant subsets $\Delta_{0}, \ldots, \Delta_{d-1}$. For each $(+n,+m)$-invariant subset there is a unique way to organize its skeleton into a cycle, so that on each edge as we go along the cycle we either add $m$ or subtract $n$ (basically, one should simply follow the boundary of the corresponding Young diagram). Consider the cycle corresponding to $\Delta_{0}$. We know that $0=\min \left(\Delta_{0}\right)$ is an $n$-generator and $-m$ is an $m$-cogenerator. Let us remove the $(+m)$-edge $-m \rightarrow 0$ from the cycle, so that we get a simple path which starts from 0 and ends at $-m$. We will start from this path and then insert the cycles corresponding to $\Delta_{1}, \ldots, \Delta_{d-1}$ into it, so that in the end we get a path from 0 to $-m$ which traverses $M(-n)$-edges and $N-1(+m)$ edges. The rule is very simple: on $k$ th move we insert the cycle corresponding to $\Delta_{k}$ in the last possible position, i.e. we go along the path constructed so far till its last intersection with the cycle corresponding to $\Delta_{k}$, then go around the cycle, and then finish the rest of the path.

The resulting path encodes the boundary of the diagram $\mathcal{D}(\Delta):$ the $(+m)$-edges correspond to vertical steps, while $(-n)$-edges correspond to horizontal steps, plus there is an extra vertical step in the very beginning. In fact, as we see in the example below, this path gives us the corresponding ranks of the steps in the boundary path.

Example 2.12 Consider Example 2.11 The skeleton of $\Delta_{0}$ is $-2,0,1,2,4$. The corresponding path is

$$
0 \rightarrow 2 \rightarrow 4 \rightarrow 1 \rightarrow-2 \text {. }
$$

The cycle corresponding to $\Delta_{1}$ is

$$
0 \rightarrow 2 \rightarrow-1 \rightarrow 1 \rightarrow-2 \rightarrow 0 .
$$

The last intersection of the path with this cycle is at -2 , so after merging one gets the path

$$
0 \rightarrow 2 \rightarrow 4 \rightarrow 1 \rightarrow-2 \rightarrow 0 \rightarrow 2 \rightarrow-1 \rightarrow 1 \rightarrow-2 .
$$

The cycle corresponding to $\Delta_{2}$ is

$$
6 \rightarrow 8 \rightarrow 5 \rightarrow 7 \rightarrow 4 \rightarrow 6 .
$$

The last intersection of the new path with this cycle is at 4 , so the resulting path is

$$
0 \rightarrow 2 \rightarrow 4 \rightarrow 6 \rightarrow 8 \rightarrow 5 \rightarrow 7 \rightarrow 4 \rightarrow 1 \rightarrow-2 \rightarrow 0 \rightarrow 2 \rightarrow-1 \rightarrow 1 \rightarrow-2 .
$$

Finally, the boundary path of $\mathcal{D}(\Delta)$ is vvvvvhvhhhvvhvh (see Figure 5 ). Note that in this example one could first merge $\Delta_{2}$ and then $\Delta_{1}$ with the same result. This is because their skeletons do not intersect. 
Similar to the $d=1$ case, the steps of the boundary path correspond to the $N$-generators and $M$ cogenerators of $\Delta$. However, the correspondence is a bit trickier. The label in the box above a horizontal step (correspondingly, to the left of a vertical step) is equal to $\left\lfloor\frac{x}{d}\right\rfloor$, where $x$ is the $M$-cogenerator ( Ngenerator).

Theorem 2.13 The map $\mathcal{D}: \mathbf{M}_{N, M} / \sim \longrightarrow Y_{N, M}$ is a bijection.

Sketch of the proof. Given a diagram $D \in Y_{N, M}$, all we need to do to recover the corresponding minimal $(+N,+M)$-invariant subset is to assign the remainders modulo $d$ to the steps of the boundary path of $D$. This is done by reverting the gluing procedure as follows. Find the lowest $m+n$ consecutive steps in the boundary path of $D$, whose endpoints differ by $\overrightarrow{(m,-n)}$. Color these steps $d-1$. Then remove this part of the path, and shift the upper part of the remaining steps by $\overrightarrow{(m,-n)}$ to make the remaining path connected. Repeat this procedure, using the color $d-k$ at the $k$-th iteration (see Figure 5 for an example).

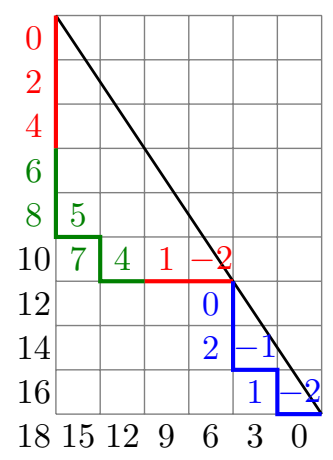

Fig. 5: $\mathbb{Z} / 3 \mathbb{Z}$-coloring of a $(9,6)$ Dyck path

The following theorem now follows from the construction.

Theorem 2.14 The sweep map $\zeta: Y_{N, M} \rightarrow Y_{N, M}$ factorizes according to Figure 2

$$
\zeta=\mathcal{G} \circ \mathcal{D}^{-1}
$$

for all positive $N, M$.

\section{References}

[1] J. Anderson. Partitions which are simultaneously $t_{1}$ - and $t_{2}$-core. Discrete Math. 248 (1-3) (2002). 237-243.

[2] D. Armstrong. Hyperplane arrangements and diagonal harmonics. 23rd International Conference on Formal Power Series and Algebraic Combinatorics (FPSAC 2011), 39-50, Discrete Math. Theor. Comput. Sci. Proc., AO, Assoc. Discrete Math. Theor. Comput. Sci., Nancy, 2011. 
[3] D. Armstrong, N. Loehr, G. Warrington, Sweep maps: A continuous family of sorting algorithms, arXiv:1406.1196.

[4] A. Beauville, Counting rational curves on K3-surfaces. Duke Math. J. 97 (1999), 99-108.

[5] F. Bergeron, A. Garsia, E. Leven, G. Xin, Compositional $(k m, k n)$-Shuffle Conjectures. arXiv:1404.4616 [math.CO].

[6] C. Ceballos, T. Denton, C. R. H. Hanusa, Combinatorics of the zeta map on rational Dyck paths, to appear in Journal of Combinatorial Theory, series A, arXiv:1504.06383.

[7] E. Gorsky, M. Mazin. Compactified Jacobians and $q, t$-Catalan Numbers, I. Journal of Combinatorial Theory, Series A 120 (2013), pp. 49-63.

[8] E. Gorsky, M. Mazin. Compactified Jacobians and $q, t$-Catalan numbers, II. J. Algebraic Combin. 39 (2014), no. 1, pp. 153-186.

[9] E. Gorsky, M. Mazin, M. Vazirani, Affine Permutations And Rational Parking Functions, accepted in Transactions of American Mathematical Society, arXiv:1403.0303.

[10] J. Haglund, The $q, t$-Catalan Numbers and the Space of Diagonal Harmonics: With an Appendix on the Combinatorics of Macdonald Polynomials. AMS University lecture series, 2008.

[11] N. Loehr. Conjectured Statistics for the Higher $q, t$-Catalan Sequences. The Electronic Journal of Combinatorics 12 (2005), Research Paper 9, 54 pp.

[12] N. Loehr, G. Warrington. A continuous family of partition statistics equidistributed with length. Journal of Combinatorial Theory, Series A 116 (2009), 379-403.

[13] G. Lusztig, J. M. Smelt. Fixed point varieties on the space of lattices. Bull. London Math. Soc. 23 (1991), no. 3, 213-218.

[14] M. Mazin, A bijective proof of Loehr-Warrington's formulas for statistics $\operatorname{ctot} \frac{q}{p}$ and $\operatorname{mid} \frac{q}{p}$, Ann. Comb. 18 (2014), no. 4, pp. 709-722.

[15] J. Piontkowski, Topology of the compactified Jacobians of singular curves. Math. Z. 255 (2007), no. 1, 195-226.

[16] N. Williams, Sweeping up zeta, arXiv:1512.01483.

[17] G. Xin, An efficient search algorithm for inverting the sweep map on rational Dyck paths, arXiv:1505.00823. 